management of refractory ascites. We conducted a retrospective analysis of a large series of patients undergoing TIPS insertion for this indication.

Aim The aims of this study were to describe the series of patients undergoing TIPS insertion for refractory ascites at the Royal Free Hospital particularly with regards to survival and procedural success

Method A retrospective analysis of the Royal Free Hospital radiology database was conducted to identify all patients who underwent all TIPS procedures between January 1991 and January 2011. Patient records were used to subsequently identify those patients in whom refractory ascites was the principal indication for TIPS insertion and to characterise this patient cohort. Patients were excluded if hydrothorax was the primary indication for TIPS insertion. All patients underwent baseline EEG/echocardiography and cross sectional imaging as part of their pre-procedural work up. Patients were requiring regular paracentesis and were either diuretic insensitive or intolerant.

Results 1073 TIPS-related procedures were conducted at the Royal Free Hospital between January 1991 and January 2011. Of these, 159 patients underwent TIPS insertion for refractory ascites. Within this patients cohort, the underlying aetiology of cirrhosis was: alcohol $56.0 \%$ (89/159), hepatitis C $12.6 \%$ (20/159), cryptogenic 8.8\%(14/ $159)$ and other causes $22.6 \%$ (36/159). 29\% (46/159) of the patients were female, $71 \%(113 / 159)$ were male. The mean age at the time of TIPS insertion was $54.3( \pm 0.94)$ yrs. The mean pre-TIPS MELD score was $15.26( \pm 0.57)$ with a mean pre-TIPS EEG frequency of $7.51 \mathrm{~Hz}( \pm 0.20)$. The mean post-procedural portal pressure gradient was $11.0 \mathrm{~mm} \mathrm{Hg}$ ( \pm 0.57$)$. Six month, 12 month and 2-year survival post-TIPS insertion was $78 \%, 50 \%$ and $50 \%$ respectively. At 6 months, $63 \%$ of patients had no or minimal ascites, $29 \%$ had moderate volume ascites and only $8 \%$ had persistent large volume ascites. At 12 months, $69 \%$ of patients had no or minimal ascites, $21 \%$ had moderate volume ascites and $10 \%$ had persistent ascites requiring paracentesis.

Conclusion In a carefully selected group of patients, TIPS is an effective intervention in the management of refractory ascites.

\section{OP10 END-STAGE METHOTREXATE-ASSOCIATED CHRONIC LIVER DISEASE IN THE USA: INTERACTION OF DRUG, HOST AND ENVIRONMENTAL FACTORS}

doi:10.1136/gutjnl-2011-300857b.10

M F Dawwas, G P Aithal. Nottingham Digestive Diseases Centre: National Institute of Health Research Biomedical Research Unit, Nottingham University Hospitals NHS Trust, Nottingham, UK

Introduction Methotrexate (MTX) is an effective and widely used immunosuppressant; however, long-term therapy has been associated with steatosis, progressive hepatic fibrosis and cirrhosis. Given the similarity of the histopathological features of methotrexate-associated chronic liver disease (MTX-CLD), non-alcoholic fatty liver disease (NAFLD) and alcoholic liver disease (ALD), we hypothesised that these diseases may share a common pathogenesis. Method We analysed the diagnostic records of all individuals who have been listed for liver transplantation in the USA and reported to the Organ Procurement and Transplantation Network (OPTN) during the period 1 October 1987 and 22 May 2009 to identify those whose liver disease was deemed to have been, wholly or partly, caused by methotrexate (MTX-CLD). We compared the demographic, clinical and laboratory characteristics of adult individuals with MTX-CLD with those listed for ALD and NAFLD.

Results Among 148639 unique listings for liver transplantation, we identified 105 individuals with MTX-CLD, and these were compared with individuals listed for ALD $(\mathrm{n}=17592)$ and NAFLD $(\mathrm{n}=3259)$. Concurrent liver disease among individuals with MTX-CLD included ALD in 4.8\%, NAFLD in 7.7\%, hepatocellular carcinoma in $2.9 \%$, hepatitis $\mathrm{C}$ infection in $1 \%$ and other drug-induced liver disease in $1 \%$. Compared to the ALD group, those with MTX-CLD were older (median age 57 vs 51 years, $p<0.0001$ ), more likely to be Caucasian ( $91.4 \%$ vs $80.9 \%, \mathrm{p}<0.007$ ), female (46.2\% vs $19.2 \%$, $\mathrm{p}<0.001)$ and diabetic $(36.8 \%$ vs $18.3 \%, \mathrm{p}<0.001)$, and had a higher body mass index (median $28.2 \mathrm{vs} 27.2 \mathrm{~kg} / \mathrm{m}^{2}, \mathrm{p}<0.03$ ), but a lower median MELD score (14.5 vs $16, \mathrm{p}<0.007)$. In contrast, compared to individuals with NAFLD, those with MTX-CLD were less likely to be diabetic $(36.8 \%$ vs $47.7 \%, \mathrm{p}<0.05)$ and had a lower median body mass index ( $\left.28.2 \mathrm{vs} 32.1 \mathrm{~kg} / \mathrm{m}^{2}, \mathrm{p}<0.0001\right)$, but a similar age, gender, ethnicity and MELD distribution. The prevalence of hypertension and vascular disease did not differ among the three groups, nor did their complications (ascites, encephalopathy, spontaneous bacterial peritonitis) profile.

Conclusion This is the largest analysis of end-stage MTX-CLD reported to date, demonstrating that it is a rare form of cirrhosis that has a distinct risk factor profile from those of ALD and, to a lesser extent, NAFLD. The severity of MTX hepatotoxicity may be potentiated by host (ethnicity) and environmental (diabetes, obesity) factors, ultimately leading to decompensated disease. A common pathogenic process may underlie MTX-CLD, ALD and NAFLD

\section{OP11 ARE SIMPLE NON-INVASIVE SCORING SYSTEMS FOR FIBROSIS RELIABLE IN PATIENTS WITH NAFLD AND NORMAL ALT LEVELS?}

doi:10.1136/gutjnl-2011-300857b.11

${ }^{1} \mathrm{~S}$ McPherson, ${ }^{2} \mathrm{O}$ Anstee, ${ }^{3} \mathrm{E}$ Henderson, ${ }^{2} \mathrm{~A}$ Burt, ${ }^{2} \mathrm{C}$ Day. ${ }^{1}$ Liver Unit, Freeman Hospital, Newcastle upon Tyne; ${ }^{2}$ Institute of Cellular Medicine Newcastle University; ${ }^{3}$ Liver Unit Freeman Hospital Newcastle upon Tyne

Introduction With a quarter of the UK population estimated to have some degree of NAFLD, there is a need for a robust and economical population based screen to identify individuals with advanced fibrosis. It is recognised that many patients with NAFLD have normal-range ALT levels. Non-invasive scoring systems may exclude advanced disease without the need for liver biopsy, however reliability of these tests in this population has not been determined.

Aim To assess performance of several simple non-invasive tests for fibrosis in patients with biopsy-proven NAFLD and normal ALT levels.

Method Patients who were reviewed in the Freeman Hospital fatty liver clinic between 1999 and 2009 were included. Liver biopsies were assessed using the Kleiner score. The AST/ALT ratio, BARD, FIB-4 and NAFLD fibrosis scores were calculated from blood tests taken within 6 months of liver biopsy.

Results 305 patients were included (70 with normal ALT [ALT $<30 \mathrm{IU} / 1$ for females and ALT $<45 \mathrm{IU} / 1$ for males] and 235 with raised ALT). $24 \%$ of subjects with normal ALT and $17 \%$ with raised ALT had advanced fibrosis (Kleiner stage 3-4). Patients with normal ALT were significantly younger $(p=0.004)$ and had lower BMI $(p=0.03)$ than those with raised ALT. The area under ROC curve (AUROC), sensitivity, specificity, positive and negative predictive values for a diagnosis of advanced fibrosis using each score is shown in Abstract OP11 table 1. In addition, the proportion of patients with a score below the cut-off who would avoid liver biopsy is displayed. The specificity of the AST/ALT ratio, BARD and NAFLD fibrosis scores for advanced fibrosis was low in patients with normal ALT, which would result in a high proportion of patients with mild disease having a liver biopsy. FIB-4 score performed best overall. 
Abstract 0P11 Table 1

\begin{tabular}{lllllllll}
\hline Normal ALT [ALT <30 IU/I for females and ALT <45 IU/I for males] \\
\hline Test & AUROC & Cut-off & Sens (\%) & Spec (\%) & PPV (\%) & NPV (\%) & Avoid biopsy \\
\hline AST/ALT & 0.81 & 0.8 & 94 & 44 & 35 & 96 & $18 / 70(26 \%)$ \\
BARD & 0.71 & 2 & 94 & 26 & 29 & 93 & $15 / 70(21 \%)$ \\
FIB-4 & 0.86 & 1.30 & 82 & 77 & 52 & 92 & $43 / 70(61 \%)$ \\
NAFLD & 0.85 & -1.455 & 82 & 51 & 35 & 90 & $29 / 70(41 \%)$ \\
Raised ALT & & & & & & & \\
AST/ALT & 0.79 & 0.8 & 59 & 86 & 46 & 91 & $184 / 235(78 \%)$ \\
BARD & 0.78 & 2 & 83 & 57 & 28 & 94 & $118 / 235(50 \%)$ \\
FIB-4 & 0.85 & 1.30 & 81 & 72 & 37 & 95 & $148 / 235(63 \%)$ \\
NAFLD & 0.80 & -1.455 & 71 & 65 & 29 & 92 & $138 / 235(59 \%)$ \\
\hline
\end{tabular}

Conclusion The FIB-4 score performed well in patients with normal or raised ALT, reliably excluding advanced fibrosis and reducing the need for liver biopsy. Therefore, the FIB-4 score may be an appropriate tool for use in primary care to triage patients with NAFLD who need referral for further assessment. Further validation in a general practice cohort is underway.

\section{Viral hepatitis}

\section{OP12 SIMILAR SVR RATES IN IL28B CC, CT OR TT PRIOR RELAPSER, PARTIAL- OR NULL-RESPONDER PATIENTS TREATED WITH TELAPREVIR/PEGINTERFERON/RIBAVIRIN: RETROSPECTIVE ANALYSIS OF THE REALIZE STUDY}

doi:10.1136/gutjnl-2011-300857b.12

${ }^{1} \mathrm{G}$ R Foster, ${ }^{2} \mathrm{~S}$ Pol, ${ }^{3} \mathrm{~J}$ Aerssens, ${ }^{4} \mathrm{~S}$ Zeuzem, ${ }^{5} \mathrm{P}$ Andreone, ${ }^{6} \mathrm{E} \mathrm{J}$ Lawitz, ${ }^{7} \mathrm{~S}$ Roberts, ${ }^{8} \mathrm{Z}$ Younossi, ${ }^{9} \mathrm{R}$ Focaccia, ${ }^{10} \mathrm{~A}$ Horban, ${ }^{11} \mathrm{P} \mathrm{J}$ Pockros, ${ }^{3} \mathrm{R}$ Van Heeswijk, ${ }^{3} \mathrm{~S}$ de Meyer, ${ }^{12} \mathrm{D}$ Luo, ${ }^{3} \mathrm{M}$ Beumont, ${ }^{12} \mathrm{G}$ Picchio. ${ }^{1}$ Institute of Cell and Molecular Science, Queen Mary University of London, London, UK; ${ }^{2}$ University Paris Descartes, INSERM UnitĖ 567, and Assistance Publique-Hopitaux de Paris, Cochin Hospital, Paris, France; ${ }^{3}$ Tibotec BVBA, Beerse, Belgium; ${ }^{4}$ Johann Wolfgang Goethe University Medical Center,

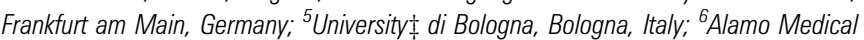
Research, Ltd, San Antonio, Texas, USA; 'Department of Gastroenterology, Alfred Hospital, Melbourne, Victoria, Australia; ${ }^{8}$ Center for Liver Disease, Inova Fairfax Hospital, Falls Church, Virginia, USA; ${ }^{9}$ Emilio Ribas Infectious Diseases Institute, $S_{\text {, }} 0$ Paulo, Brazil; ${ }^{10}$ Medical University of Warsaw, Wolska, Warsaw, Poland; ${ }^{11}$ Scripps Clinic and The Scripps Research Institute, La Jolla, California, USA; ${ }^{12}$ Tibotec Inc., Titusville, New Jersy, USA

Introduction IL28B polymorphisms are linked to differences in SVR rates in HCV treatment-naÔve patients treated with pegylated interferon (P) and ribavirin (R). REALIZE is a Phase 3 study that compared the efficacy, safety and tolerability of telaprevir $(\mathrm{T})$, with or without a Lead-in (LI), in combination with PR against PR alone in prior treatment-failure patients including relapsers, partial responders and null responders (NR). Both T/PR arms were superior to control in all three patient categories. The relationship between IL28B genotype and SVR was investigated retrospectively.

Method 527/662 (80\%) patients enrolled in REALIZE consented to genetic testing. This represented $72 \%, 76 \%$ and $98 \%$ of the total relapsers, partial responders, and NR, respectively. Genotype rs12979860 was determined using a TaqMan allelic discrimination assay validated against Sanger sequencing on 50 independent samples. This was a retrospective study based on patients who consented to genetic testing prior to the discovery of IL28B, thus, sample size was not based on formal statistical considerations.

Results Overall, 94\% were Caucasian and 4\% were Black. $18 \%$ of patients were IL28B CC, $61 \%$ CT and 21\% TT. By prior response category, the highest proportion of IL28B TT patients was among prior NR (28\%) while the highest frequency of CC patients occurred among prior relapsers (27\%). The observed IL28B genotype frequencies indicate that the population was not in Hardy-Weinberg equilibrium $\left(\chi^{2}=28, p<0.0001\right)$. IL28B genotypes were well balanced across all arms with exception of a higher frequency of TTs in the placebo arm. Since no differences were observed between the two T arms, a pooled analysis is presented.

Conclusion Differences in SVR rates among IL28B CC, CT and TT patients were only evident when the three patient subpopulations were pooled, however, SVR among CT and TT patients were still high. In this retrospective analysis, IL28B genotype did not contribute to outcome prediction in prior treatment-experienced patients treated with a telaprevir-based regimen and thus, may be of limited utility in this setting.

\section{OP13 CD8B LOW T CELLS ARE A PROMINENT, FUNCTIONALLY DISTINCT POPULATION IN CHRONIC HEPATITIS B INFECTION}

doi:10.1136/gutjnl-2011-300857b.13

${ }^{1} \mathrm{E}$ Marrinan, ${ }^{2} \mathrm{~L}$ Walker, ${ }^{3} \mathrm{E}$ Barnes, ${ }^{4} \mathrm{P}$ Klenerman. ${ }^{1}$ Peter Medawar Building for Pathogen Research, Nuffield Department of Medicine, University of Oxford; ${ }^{2}$ Peter Medawar Building for Pathogen Research; ${ }^{3}$ Nuffield Department of Medicine, ${ }^{4}$ University of Oxford

Introduction The failure of antigen-specific CD8 T cells is a well recognised feature of chronic hepatitis $\mathrm{B}(\mathrm{HBV})$ infection, however the bulk CD8 $\mathrm{T}$ cell population is also characterised by low expression of CD28 and poor IL-2 production and proliferation capacity (Das, Hoare et al 2008). We have previously observed a prominent $\mathrm{CD} 8 \mathrm{~T}$ cell population in chronic $\mathrm{HBV}$ to be $\mathrm{CD} 8 \mathrm{~b}^{\text {low }}$ (Walker, Kang et al) - this is most obvious in the cells which lack expression of CD161 (a molecule associated with liver homing). The relationship of these two observations has not previously been explored. Such changes may represent infection induced immune exhaustion or an acquired tolerance mechanism, which is breached during flares in disease activity. Both scenarios have important implications either for development of immunotherapy for viral clearance or treatment for prevention of disease progression.

Aim The aim of this study was to further characterise the phenotypic and functional features of the bulk CD8 T cell population in chronic HBV infection and the relationship of $\mathrm{CD} 288^{\text {low }} \mathrm{CD} 8+$ and CD161-CD8b ${ }^{\text {low }} \mathrm{T}$ cell populations.

Method Peripheral blood mononucleocytes were obtained from patients with eAg+ve chronic HBV ( $\mathrm{n}=6)$, eAg-ve chronic HBV $(n=12)$, chronic hepatitis C (HCV) genotype $1(n=5)$ and healthy

Abstract OP12 Table 1

\begin{tabular}{|c|c|c|c|c|c|c|c|c|}
\hline \multirow[b]{2}{*}{$\%$ SVR (n) } & \multicolumn{2}{|c|}{ Overall Population } & \multicolumn{2}{|l|}{ Prior Relapsers } & \multicolumn{2}{|c|}{ Prior Partial Responders } & \multicolumn{2}{|c|}{ Prior Null Responders } \\
\hline & $\begin{array}{l}\text { Pooled } \\
\text { T12/PR48 Arms } \\
\text { (N=422) }\end{array}$ & $\begin{array}{l}\text { Pbo/PR48 } \\
\text { Arm (N=105) }\end{array}$ & $\begin{array}{l}\text { Pooled } \\
\text { T12/PR48 Arms } \\
\text { (N=209) }\end{array}$ & $\begin{array}{l}\text { Pbo/PR48 } \\
\text { Arm }(\mathrm{N}=52)\end{array}$ & $\begin{array}{l}\text { Pooled } \\
\text { T12/PR48 Arms } \\
(\mathrm{N}=79)\end{array}$ & $\begin{array}{l}\text { Pbo/PR48 } \\
\text { Arm }(N=20)\end{array}$ & $\begin{array}{l}\text { Pooled } \\
\text { T12/PR48 Arms } \\
\text { (N=134) }\end{array}$ & $\begin{array}{l}\text { Pbo/PR48 } \\
\text { Arm (N=33) }\end{array}$ \\
\hline IL28B CC & $79(76)$ & $29(17)$ & $88(58)$ & $33(12)$ & $63(8)$ & $20(5)$ & $40(10)$ & NA (0) \\
\hline IL28B CT & $60(266)$ & $16(58)$ & $86(117)$ & $20(30)$ & $58(57)$ & $20(10)$ & $29(92)$ & $6(18)$ \\
\hline IL28B TT & $61(80)$ & $13(30)$ & 85 (34) & $30(10)$ & 71 (14) & $0(5)$ & 31 (32) & $7(15)$ \\
\hline
\end{tabular}

